\title{
Cada curso un aprendizaje-servicio
}

\section{Brenda Bär y Josep Ma Puig}

Facultad de Educación, Universidad de Barcelona

\section{Resumen}

El artículo estudia el proceso que han seguido tres centros educativos -el IES Eduard Fontseré, el centro de formación profesional Solc Nou y la Escuela de Educación Especial Xaloc- para conseguir ofrecer a todo su alumnado al menos una experiencia de aprendizaje-servicio cada curso escolar. En los tres casos la implantación masiva de la metodología del aprendizaje-servicio ha supuesto un cambio profundo en el centro que les ha permitido mejorar sus resultados e incrementar su satisfacción como docentes. Con una metodología etnográfica -entrevistas, observación y análisis de documentación- se han descrito las claves que explican cómo implantar en todos los cursos de un centro la metodología del aprendizaje-servicio.

\section{Palabras clave}

Aprendizaje-servicio, educación en valores, cultura de centro, liderazgo, equipos docentes. 


\title{
Each course a service-learning
}

\begin{abstract}
This paper discussed the trajectories followed by three educational centres - the Eduard Fontseré Secondary School, the Solc Nou vocational training centre and the Xaloc Special Needs School- in order to offer all their students at least one servicelearning experience every academic year. In all three cases, mass implementation of the service-learning methodology has led to profound changes in their centres, which have allowed them to improve their academic results and increase teacher satisfaction. Using ethnographic methods -interviews, observation and analysis of documentation - this paper discusses the key elements that are involved in successful implementation of service-learning for all students in educational centres.
\end{abstract}

\section{Keywords}

Service-learning, values education, center culture, leadership, teaching team. 


\section{Introducción y objetivos}

Hay experiencias únicas cuya huella perdura para siempre. Son acontecimientos que impactan de lleno en la construcción de la identidad. Pese a ser experiencias puntuales, quizás ocurren una sola vez, provocan hondos efectos formativos. Si lo singular $y$ único influye, lo repetido quizás tenga todavía más fuerza educativa. Adquirir competencias, fijar hábitos o formar el carácter supone repetir actividades hasta lograr su dominio. Aprender es entrenar aquello que se desea dominar.

El aprendizaje-servicio es una propuesta educativa de múltiples efectos formativos: apunta a finalidades valiosas, tales como la formación de ciudadanos participativos y proporcionar sentido cívico al saber escolar (Barber, 2000; Dewey, 1967), mejora los resultados académicos (Furco, 2004) y produce una honda satisfacción en los participantes (Folgueiras, Luna y Puig, 2013).

Si participar en actividades de aprendizaje-servicio produce tantos beneficios, implicarse de manera repetida probablemente contribuirá en mayor medida a alcanzar dichos logros. Suponemos que participar a menudo en actividades de aprendizaje-servicio facilita una mejor adquisición de valores, una formación personal y académica más completa $y$, finalmente, va a dejar en el alumnado un recuerdo y una motivación favorables a este tipo de actividades. Consideramos pues muy conveniente multiplicar las propuestas de aprendizaje-servicio que los centros proponen a su alumnado.
Sin embargo, esta no es a día de hoy la situación más habitual. Los centros educativos suelen ofrecer actividades de aprendizaje-servicio en una sola ocasión durante toda la escolaridad, o en el mejor de los casos unas pocas veces. De acuerdo con nuestra suposición, lo mejor sería ofrecer con frecuencia actividades de aprendizajeservicio, quizás lo más idóneo fuese una vez por curso académico. De este modo el aprendizaje-servicio sería una seña de identidad del centro, formaría parte del núcleo de su línea educativa y se presentaría al alumnado como una forma habitual de trabajo.

Tal como hemos advertido, hay muchos centros que realizan una actividad de aprendizaje-servicio y muy pocos que lo hayan convertido en línea del centro y lo ofrezcan cada curso a su alumnado. Por lo tanto, el objetivo de esta investigación es ver qué se debe hacer para lograr que un centro educativo ofrezca anualmente actividades de aprendizaje-servicio a todo su alumnado. Para responder a este interrogante hemos estudiado algunos centros que durante los últimos años han pasado de no conocer el aprendizaje-servicio a ofrecerlo cada curso a todo su alumnado. Se trata de centros que han realizado un trabajo excelente y que pueden ayudarnos a responder a los interrogantes que han orientado la investigación:

- Qué fases atraviesan los procesos de implantación del aprendizajeservicio a todos los niveles y grupos de un centro

- Qué tipo de liderazgo y de trabajo en equipo facilita la generalización 


\section{del aprendizaje-servicio}

- Qué reflexiones pedagógicas supone la programación masiva de actividades de aprendizaje-servicio

- Qué resultados produce la implementación del aprendizajeservicio a todo un centro educativo

- Que recomendaciones pueden hacerse a un equipo docente que desee generalizar el aprendizajeservicio en su centro

\section{Metodología}

El estudio que presentamos busca entender lo ocurrido en los diferentes centros desde la perspectiva de sus propios participantes. Para conseguirlo se propone una aproximación etnográfica, al estilo de lo que Taylor y Bogdan (1987) entienden como un enfoque fenomenológico de la investigación y para el cual recomiendan métodos cualitativos con los que conseguir una comprensión de los fenómenos sociales desde la perspectiva de quien los vive en primera persona conociendo $y$ valorando su marco de referencia. De acuerdo con tal postura, se han usado como instrumentos la entrevista en profundidad y la observación participante. Sumadas a estas dos técnicas, también se ha hecho una consulta de diferentes fuentes de documentación sobre los centros educativos, tales como Proyectos Educativos de Centro anteriores y actuales, información de las páginas web y noticias publicadas en ellas, artículos y estudios académicos sobre estos centros, datos de matriculación, cualificaciones, asistencia y otra información intenta de centro.

Los centros escogidos para llevar a cabo la experiencia fueron seleccionados por ser aquellos donde se realizan diversos proyectos de aprendizaje-servicio y al menos todo el alumnado pasa por uno de ellos durante el curso. No abundan este tipo de casos en Cataluña y dentro de los existentes se seleccionaron tres colegios siguiendo el criterio principal de la reconocida calidad en sus actuaciones y la posibilidad de acceso a los mismos. También se tuvo en cuenta que cubrieran diferentes tipos de etapas educativas y que hubiera algún ejemplo tanto de titularidad pública como de titularidad concertada. Siguiendo estos criterios tuvimos acceso a los siguientes centros de diferentes maneras:

\section{Instituto público de educación} secundaria Eduard Fontserè del barrio de la Florida en Hospitalet de Llobregat. Cuenta con más de 400 alumnos y alumnas de secundaria, bachillerato y ciclos formativos. A partir del año 2004, fue de los primeros en apostar por este tipo de proyectos en la ciudad. En el pudimos realizar siete entrevistas: cinco a quien fuera en director del centro, una a la profesora implicada en diversos proyectos y otra al integrador social, figura relevante en los proyectos y la transformación del centro. Además se nos facilitó diversa información y documentación interna del centro, así como otros artículos y datos de estudios académicos realizados sobre el centro (Badia y Longas, 2013; Folgueiras, Luna y Puig, 2013). 
Escuela de Educación Especial Xaloc de Sabadell. Se trata de un centro concertado que se inició como una cooperativa de familias y profesorado y que cubre diferentes epatas educativas desde infantil hasta postobligatoria. En el año 2001 crean una etapa postobligatoria para alumnado entre los 16 y los 20 años, etapa en la que nos centraremos. En este centro se grabaron más de diez horas de entrevista al psicólogo referente de la etapa 16-20, muy activo en la creación de la etapa e impulsor de su enfoque hacia el aprendizaje-servicio, y que también fue director del centro durante algunos años de la formación de esta etapa. También se realizaron dos entrevistas a personas referentes de TAINA y ANDI, dos organizaciones de inserción laboral y social donde acuden estudiantes posteriormente a su paso por el centro. Además se tuvo acceso a diversa documentación interna del centro y se realizó una visita para conocer al profesorado y alumnado.

Colegio Solc Nou de Barcelona. Es una escuela concertada dedicada exclusivamente a ciclos formativos, entre los cuales ofrece dos de nivel medio (Cuidados Auxiliares de Enfermería y Farmacia y Parafarmacia) y uno de nivel superior (Técnico de Educación infantil) a 150 alumnos y alumnas con un claustro compuesto por 20 profesionales. Desde hace más de 10 años que la escuela realiza proyectos de aprendizaje-servicio y actualmente han conseguido que todo su alumnado pase por alguno durante cada curso escolar. Dada la riqueza de esta experiencia, además de las entrevistas -siete a miembros del equipo directivo y seis a las coordinadoras de las diferentes áreas-, tuvimos acceso al centro durante un curso para realizar observaciones en las diferentes clases donde se llevaba a cabo proyectos de aprendizaje-servicio, reuniones de equipo y claustros.

También como en las otras experiencias pudimos acceder a diversa documentación interna del centro y artículos de tipo divulgativo sobre sus experiencias.

\section{Etapas en la generalización del Aps en un centro}

Con la primera pregunta de investigación pretendemos conocer los pasos que ha seguido la incorporación masiva del aprendizaje-servicio en los centros estudiados. Se quieren conocer dos cuestiones: las fases a través de las cuales se ha implantado el aprendizaje-servicio y si estas etapas son parecidas en todos los centros analizados. A partir de la comparación del material obtenido durante el trabajo de campo y de los primeros escritos descriptivos de los procesos de cada uno de los centros (Bär, 2016a, 2016b; Carmona, Bär y Puig, 2016), hemos podido establecer una sucesión de cinco pasos, que a continuación vamos a presentar.

Del problema al reto. En todos los casos estudiados, los centros se han movilizado buscando una mejora urgente tras constatar que tenían un problema que precisaba cambios drásticos. Siempre desde el marco singular de la situación de cada centro, se encuentra una evidente situación de crisis que no es posible evitar ni olvidar, 
sino que conviene tomar conciencia de sus dimensiones $y$, sobre todo, definirla con la mayor precisión posible. La correcta definición de los rasgos negativos de la situación es fundamental porque al positivarlos empieza a perfilarse la imagen del reto que el centro debe asumir.

\begin{abstract}
En Solc Nou se habla de fatiga institucional para referirse al desgaste de los modos habituales de trabajar y al desconcierto provocado por ciertos cambios legales y del contexto que hacer que se tambalee el sentido de su trabajo formativo. Perdida de sentido que se expresa en el miedo a transmitir una profesión sin valores, a la dificultad en implicar al alumnado en la vida y el trabajo del centro y a la necesidad de construir un auténtico proyecto de centro para toda la escuela y el alumnado de todos sus turnos. (Carmona, Bär y Puig, 2016, 18-19)
\end{abstract}

L'IES Fontseré tenía la etiqueta de centro a evitar por sus malos resultados, la tasa muy alta de abandono, el absentismo agudo, la conflictividad entre el alumnado y con el profesorado y, en definitiva, una mala imagen social que había provocado un descenso alarmante de la matrícula. (Bär, 2016a, 5-8)

EEE Xaloc quiere ser un centro que integre a su alumnado y evite que al terminar la escolaridad vuelvan a su casa y se encierren incapaces de valerse por sí mismos. Sin embargo, pronto se dan cuenta que es imposible conseguirlo escolarizando tan solo hasta los dieciséis años. Conviene prolongar la formación para conseguir unas mejores competencias para la vida autónoma y la actividad laboral.

(Bär, 2016b, 5-11)

Alerta activa. A partir del reto que exigen los problemas que aquejan al centro, se abre una segunda etapa que hemos calificado de alerta activa porque es como si las personas que impulsan el cambio desplegaran sus antenas en busca de elementos que les ayuden a trazar la transformación que necesita el centro. Este radar que busca propuestas para superar los problemas, en todos los casos estudiados se ha orientado hacia tres direcciones complementarias: la primera, en busca de aciertos producidos por acciones realizadas en el mismo centro para hacer frente a los problemas detectados; en segundo lugar, en todos los casos ha resultado muy importante el encuentro y la cooperación con entidades de la comunidad; y en tercer lugar, el hallazgo más o menos casual del aprendizaje-servicio, pero que siempre ha ido seguido del acierto intencional de reconocer que esta metodología podía ser una muy buena herramienta para hacer frente a los problemas del centro. El resultado de esta etapa de alerta y búsqueda ha permitido iniciar pruebas tentativas, como veremos en el siguiente paso. Ahora describiremos brevemente uno de los casos estudiados, aunque tal como se ha dicho en los tres se dieron procesos semejantes.

En el caso del IES Eduard Fontseré el nuevo equipo directivo tuvo que empezar mostrando a todos que las normas se deben cumplir, que es posible crear un clima de confianza y que cada uno de los alumnos es una persona importante para el profesorado. Este trabajo interno se completo con un segundo elemento esencial: la obtención de una ayuda del plan PROA, una contribución que les obligó a trazar un plan estratégico y definir un conjunto de mejoras y de acciones para alcanzarlas. En este nuevo horizonte de transformaciones, conectaron también 
con una entidad social que permitió vincular el centro con la comunidad. El club de tiempo libre La Florida se convirtió en un socio con quien compartir espacios, desarrollar proyectos $y$, finalmente, descubrir juntos el aprendizaje servicio e idear el primer proyecto.

(Bär, 2016a, 8-13)

\section{Primera tentativa. Parece como si} incluso las más amplias transformaciones de un centro tuvieran que empezar siempre con una pequeña experiencias que, si sale bien, da confianza a sus impulsores, les permite repetirla y mejorarla y, finalmente, generalizarla. Así ha ocurrido en los tres procesos estudiados, la etapa de alerta activa ha servido para orientarse, recoger ideas, ensayar propuestas y acabar reconociendo el valor del aprendizaje-servicio. Sin embargo, el conocimiento de esta nueva metodología y a menudo el darse cuenta de la semejanza con actividades que ya se realizaban en el centro, es solo la antesala de la realización de una primera prueba que, tras su evaluación y éxito, se decide continuar y generalizar. En las situaciones estudiadas, el aprendizajeservicio se ha ensayado con una tentativa, cuyos resultados positivos sirven para darle continuidad y pensar en nuevas posibilidades.

Siguiendo con la historia del IES Eduard Fontseré merece la pena constatar que las primeras pruebas no fueron un éxito y que solo su persistencia les permitió alcanzar los primeros logros. Al principio, en colaboración con la Associació de Veïns de La Florida se organizó la actividad Lectores para la personas mayores, que ofrecía la posibilidad de leer a personas mayores que no se podían valer por sí mismas. El proyecto no prosperó. Un segundo intento, esta vez realizado con éxito, se organizó en colaboración con el Club de tiempo libre de La Florida. El proyecto Joves pel barri consiste en ofrecer al alumnado del instituto, tras una formación adecuada, la posibilidad de contribuir como ayudante de monitor en las actividades del Club. El proyecto ha acabado convirtiéndose en bandera del centro y se ha generalizado por toda ciudad de l'Hospitalet.

(Bär, 2016a, 13-16)

De la novedad a la innovación. En todos los casos analizados, las actividades de aprendizaje-servicio empezaron como ideas nuevas, tentativas y originales del centro para intentar dar respuesta a los retos que tenían planteados. Sin embargo, tras las primeras pruebas desarrolladas con éxito, la propuesta del aprendizajeservicio pronto se fue convirtiendo en una verdadera innovación institucional, en una idea de éxito que se difundió y fue impregnando lentamente la manera habitual de llevar a cabo la misión formativa particular de cada uno de los centros. El proceso de innovación que desencadenó la confianza en la metodología del aprendizaje-servicio supuso emprender tres tareas entrelazadas: por una parte, involucrar a la mayor parte del profesorado de cada una de los centros; en segundo lugar idear y organizar nuevos proyectos de aprendizaje-servicio y, en tercer lugar, generalizar sus beneficios hasta alcanzar a todo el alumnado del centro al menos una vez cada curso escolar. Estas tareas no solo requieren una notable competencia pedagógica para idear y poner en marcha múltiples proyectos, sino también una alta capacidad de liderazgo y de trabajo en equipo para motivar y realizar el 
volumen de trabajo y, sobre todo, de cambios que supone.

En la escuela Soc Nou, la generalización del aprendizaje-servicio empezó justo después de la primera aplicación del proyecto Cuidemos a nuestros ancianos. La experiencia se consolidó y amplió gracias a la muy buena acogida que tuvo entre el alumnado y también entre el profesorado. El alumnado pedía participar en este nuevo tipo de actividad y el profesorado empezó a ver que la actividad era bien recibida, que disminuía la resistencia de los alumnos, que aprendían con nueva motivación, que se entregaban con ganas a realizar la propuesta que se les ofrecía. Una actitud positiva que ya habían olvidado y que les impulsó a extender las experiencias de aprendizaje servicio a todos sus ciclos formativos.

(Carmona, Bär, Puig, 2016, 20-21)

Catalizador de cambios. Para los centros estudiados, el aprendizajeservicio ha sido una innovación, pero ha sido bastante más: para el profesorado ha sido un catalizador que lo ha empoderado y para el centro una plataforma segura que ha permitido impulsar otros cambios educativos relevantes. En mayor o menor medida, nuestros ejemplos muestran hasta qué punto el aprendizaje-servicio ha servido para movilizar nuevas iniciativas.

Aunque los procesos nunca se cierran y los centros que nos han ocupado siguen sus trayectos, podemos dar por terminado en este punto el proceso de implantación generalizada del aprendizaje-servicio.

Como indicábamos al iniciar este recorrido a través del proceso de implantación del aprendizaje servicio en la escuela Solc Nou, el profesorado lo considera y lo califica a menudo como de motorcillo que ha puesto en marcha en el centro otros cambios educativos importantes. Una opinión que invita a preguntarse por qué motivos atribuir ese papel catalizador al aprendizajeservicio. Parece que el aprendizajeservicio ha facilitado estos cambios por dos motivos: primero, porque se ha convertido en una experiencia de éxito para el profesorado que les ha dado motivación, fuerzas e iniciativa para seguir innovando en múltiples direcciones. El segundo motivo tiene que ver con el camino pedagógico que muestra el aprendizaje-servicio y que en cierto modo invita con insistencia a proseguir. Quien ha visto los valores encarnados en los proyectos realizados por el alumnado no puede quedar anclado en un tipo de clases de meramente explicativas. Quien ha organizado proyectos de aprendizajeservicio incorporando conocimientos de varios módulos docentes y ha logrado usar este conocimiento de modo útil y altruista tampoco puede volver, como si nada hubiese ocurrido, a las lecciones verbales y memorísticas.

(Carmona, Bär y Puig, 2016, 21-22, 101-115)

\section{Generar equipo, proyecto y liderar el cambio}

Cuando algo falla en los centros educativos se nota en el ánimo de sus profesionales y de su alumnado. Con esta investigación buscamos estudiar de qué manera estos centros consiguieron afianzar un nuevo liderazgo, rehacer el equipo docente, establecer mecanismos para idear conjuntamente un mejor proyecto formativo, aprender a compartir las responsabilidades, establecer dinámicas para un nuevo modo de operar $y$, finalmente, permanecer en conexión con las posibilidades que ofrece el entorno, así como con sus necesidades. 
Cada una de los experiencias de cambio ha tenido sus peculiaridades, que pueden encontrarse bien descritas en diferentes documentos (Bär, 2016a; Bär, 2016b y Carmona, Bär y Puig, 2017), en este apartado destacaremos los puntos en común de todos los procesos y mencionaremos las estrategias que han sido de utilidad para llevarlos a cabo.

Ninguna de las experiencias analizadas ha sido igual, ni tampoco ha seguido un proceso lineal, sino que en cada caso se han combinado iniciativas que interrelacionadas han provocado cambios significativos. De todas maneras, siempre se partía de un cierto desánimo colectivo o de un sentimiento de frustración por no poder realizar mejor la tarea que tenían encomendada. Malestar que impregnaba el centro en todas sus dimensiones.

Es como si los problemas se metieran
bajo la piel del claustro y de cada una
de las personas que lo forman La crisis
de la institución se convierte también en
una crisis del equipo y de sus
integrantes La dificultad para que la
escuela siga ejerciendo con éxito la
función educativa para la que fue creada
se traduce en conductas personales de
inseguridad, desanimo, reactividad,
inmovilismo, opiniones muy
discrepantes y enquistadas, división
entre grupos, poca disposición a la
formación y escasa confianza en las
posibilidades de cambiar Una crisis
también del equipo y del profesorado
Algo de eso aquejaba a Solc Nou Hacen
falta personas o equipo que lo pueda
asumir, con cierto apoyo en el grupo y
saber escoger el momento.
(Carmona, Bär, Puig: 2016)

De aquí la importancia de introducir innovaciones, que a la vez sirvan para devolver el ánimo al profesorado y generar cohesión de grupo. Diversos factores han conseguido dar un giro y generar una transformación en cada uno de los centros. Mejorar su funcionamiento y resultados, empoderar a los equipos docentes y trazar un proyecto compartido depende de una nueva manera de entender el liderazgo, la participación y la utilización de estrategias adecuadas a estos fines.

\section{Liderazgo para el cambio: liderar sirviendo.}

En los diversos procesos observados siempre se ha dado un cambio global que va más allá de una persona, todas las personas se acaban viendo envueltas de una u otra manera. Pero todo proceso de cambio tiene un inicio y en todos ellos hemos podido observar un liderazgo personal fuerte, que rápidamente se ha distribuido y ha adoptado una manera de ejercerse de la cual podemos extraer diversos aprendizajes. Siempre se ha formado un equipo de dirección que ha tenido una intención clara de cambio, de asumir retos y atreverse a tomar decisiones para afrontarlos. En los tres casos se ha tratado de personas que formaban parte de los equipos docentes, que conocían el centro y que no perdían relación con su día a día. Grupos que han sabido trabajar en equipo y compartir las preocupaciones cotidianas. Ha sido una manera de ejercer el liderazgo sirviendo al grupo. Apuntamos algunas de las herramientas útiles destacadas para ejercer este tipo de liderazgo: 
- Aceptar y respetar las ideas y ritmos de todas las personas

- Tener la valentía para asumir los retos y tomar decisiones

- Explicar los retos y generar ilusión por el cambio

- Comunicar con sinceridad y claridad

- Mantener una visión a medio y largo plazo y planificar el futuro

- Reconocer las propuestas surgidas del equipo

- Mantener un actitud de cuidado de las personas y de las relaciones

\section{Construir un nosotros: somos un equipo.}

Para iniciar los procesos de cambio hace falta un liderazgo que lo impulse, pero también un equipo cohesionado que lo asuma. Por eso es importante construir 0 incluso reconstruir este equipo y que se perciba otra vez como un nosotros. En los diferentes procesos observados esto se ha conseguido aceptando a todas las personas y generando espacios de reflexión para definir lo qué queremos ser juntos. Para generar este equipo comprometido ha sido imprescindible sumar todas las voluntades y aprovechar las sinergias de lo que cada persona podía aportar. Destacamos algunas herramientas e ideas:

- Reconocer a todos los miembros del equipo

- Reconocer que no siempre los objetivos son compartidos y que hay que contagiarlos

- Crear un ambiente de confianza para hablar

- Mejorar el ambiente de trabajo y pacificar el centro
- Implicar al equipo en una tarea de reflexión profunda

- Dar tiempo de maduración al equipo

Pensar a fondo un proyecto común: saber adónde vamos.

Un elemento clave de los procesos de transformación observados fue contar con un proyecto formativo adecuado a las necesidades del alumnado. En consecuencia, resulta esencial contar con espacios donde este proyecto se pueda idear. Un equipo con un buen liderazgo sólo se podrá en marcha si sabe hacia dónde se dirige, que proyecto colectivo quieren llevar a cabo. En realidad, se trata de un circuito, ya que la misma implicación en dar vida al proyecto, da identidad al equipo, lo cohesiona y otorga autoridad al liderazgo.

Siempre se ha tratado de una reflexión para construir un proyecto que ha ido más allá de la búsqueda de innovaciones metodológicas, a menudo poco conectadas y superpuestas, y ha buscado replantearse globalmente qué tipo de educación construir juntos. En definitiva, pensar la filosofía educativa del centro, para luego hacer el paso de transformar estas ideas en acciones metodológicas concretas, donde el aprendizaje-servicio siempre tuvo un papel clave. A continuación destacamos algunas herramientas y aprendizajes:

- Toma de conciencia de las dificultades del centro y construir un reto conjunto

- Abrir espacios para una reflexión conjunta

- Crear un grupo motor que dinamice el proceso 
- Desarrollar conceptos, visiones y valores compartidos

- Definir un proyecto y convertirlo en propuestas prácticas

- Ofrecer formación, recursos y buscar ayudas externas

- Evaluar las realizaciones y considerar que el error es parte del proceso

Compartir responsabilidades: los proyectos son de todos/as.

Transformaciones de este calado, no pueden llevarse a cabo por pocas personas y menos cuando se esperan que sean sostenibles y crezcan en el tiempo. Por ello todos los procesos tenían claro que era necesario un liderazgo distribuido, donde cada vez se dote al equipo de mayores responsabilidades y se sumen más personas a los proyectos. Para que el peso de la gestión comenzara a ser asumido por más personas del equipo, era necesario acompañar y dar a estas personas las herramientas para asumir tareas de responsabilidad y poder hacerlo con garantías de éxito, así como proponer rotaciones para sumar cada vez más personas al proceso. A continuación destacamos diferentes aprendizajes de este aspecto de las experiencias:

- Repartir responsabilidades entre los miembros del equipo

- Generar espacios de participación y equipos con capacidad de decisión

- Acompañar y capacitar para asumir responsabilidades

- Dar facilidades para iniciar nuevos proyecto o ideas
- Dar respondabilidades de manera rotativa

- Celebrar, reconocer y difundir los logros con el equipo

Potenciar una participación activa del entorno: educar con el barrio.

Los proyectos de aprendizaje-servicio nos obligan a colaborar con otros agentes y los centros van tomando consciencia de que el cambio real no puede llevarse a cabo sin considerar al resto de actores del entorno. En todos los procesos observados, aunque de diferente manera, destaca una voluntad de ir más allá del propio centro fomentando la implicación de todos los agentes: alumnado, familias y entidades $\mathrm{u}$ otros agentes del barrio (tiendas, centros de salud, servicios municipales, etc). Compartimos algunas ideas útiles en las experiencias:

- Asignar tareas que faciliten la participación del alumnado

- Fomentar la participación de las familias

- Conocer a las entidades del entorno y pensar posibilidades de colaboración

- Aprovechar recursos del entorno y vincularlos al proyecto de centro

- Cuidar las relaciones de partenariado con los agentes del entorno

- Potenciar el trabajo en red y crear estructuras de coordinación

- Aumentar el tiempo educativo-cívico del alumnado

- Vincularse a redes de aprendizaje servicio para compartir aprendizajes y recursos 
Dinámicas para las nuevas ideas: hacer visible el cambio.

Un cambio de cultura de centro de esta magnitud necesita que los participantes puedan visibilizar las transformaciones, experimentar nuevas dinámicas y sentirse motivados por las novedades (Carmona, Bär y Puig, 2016). Para conseguirlo se hace necesaria la ayuda de elementos, experiencias y dinámicas que ayuden a despojarse de viejas conductas y adquirir otras nuevas. En los casos considerados, advertimos la utilidad de las acciones simbólicas para crear compromisos emocionales y desarrollar nuevos modelos mentales. Por poner algunos ejemplos, en un centro se decidió remodelar los espacios, generando lugares abiertos, colaborativos y donde el equipo directivo se pudiera encontrar más fácilmente y de manera más transparente con el resto de docentes. En otros casos, se crearon eventos simbólicos o se suprimió la jornada de puertas abiertas para abrir el centro siempre que fuese necesario. En este sentido también hemos detectado el uso de elementos comunicativos, como posters o tableros de noticias, para compartir información, reforzar las nuevas prioridades y dar valor a las ideas que inspiran la nueva visión educativa. Diversas pueden ser las formas en las que cristalicen o se transmitan los cambios, pero es importante recordar que deben apoyar transformaciones reales, de nada serviría este simbolismo si estuviera vacío de un trasfondo real.

\section{Cuestiones pedagógicas}

La tercera pregunta de investigación que nos planteamos pretende conocer las problemáticas pedagógicas recurrentes y de mayor relieve que surgen en los centros educativos cuando quieren generalizar a todo su alumnado las prácticas de aprendizajeservicio. Pese a la diversidad de centros analizados y probablemente a causa de la naturaleza del aprendizaje-servicio, las cuestiones pedagógicas destacadas son prácticamente las mismas, aunque naturalmente la solución que se encuentra es distinta en cada uno de los casos considerados. Antes de presentar las cuestiones pedagógicas, veremos el listado de proyectos que ha puesto en marcha cada centro.

Los tres centros han seguido un proceso semejante de implantación del aprendizaje-servicio, un recorrido que ha pasado del desconocimiento hasta poder ofrecerlo cada curso a todo su alumnado. Convertir el aprendizajeservicio en línea del centro obliga a mantenerse en estado de alerta para programar únicamente aquellas propuestas que presentan las mejores posibilidades formativas. El resultado de este proceso ha llevado a los tres centros a ofrecer una amplia y variada gama de proyectos que resumimos en el cuadro siguiente (Bär, 2016a, 16-25; Bär, 2016b, 16-29; Carmona, Bär y Puig, 2016, 49-81). 


\begin{tabular}{|c|c|c|}
\hline $\begin{array}{l}\text { Escola Solc } \\
\text { Nou }\end{array}$ & E.E.E. Xaloc & $\begin{array}{l}\text { INS. Eduard } \\
\text { Fontseré }\end{array}$ \\
\hline $\begin{array}{l}\text { Cuidemos a } \\
\text { nuestros } \\
\text { abuelos } \\
\text { Cada día es } \\
\text { una historia } \\
\text { Donación de } \\
\text { Sangre } \\
\text { Farmaconsejos } \\
\text { BiblioArte } \\
\text { Mil y una } \\
\text { fiestas en una } \\
\text { maleta } \\
\text { Cuentos y } \\
\text { Títeres } \\
\text { 112, Salvemos }\end{array}$ & $\begin{array}{l}\text { Catering } \\
\text { solidario } \\
\text { Talleres de } \\
\text { conversación } \\
\text { con personas } \\
\text { mayores } \\
\text { Que reviva el } \\
\text { rio Ripoll } \\
\text { Lavar y } \\
\text { planchar ropa } \\
\text { para Cáritas } \\
\text { Cuenta } \\
\text { cuentos } \\
\text { El huerto de } \\
\text { las amistades } \\
\text { Maratón de } \\
\text { TV3 } \\
\text { Patrimonio al } \\
\text { descubierto } \\
\text { Arreglos y } \\
\text { mantenimiento } \\
\text { Servicio de } \\
\text { compra } \\
\text { Reciclar } \\
\text { Plastificar } \\
\text { Rincón de Mari }\end{array}$ & $\begin{array}{l}\text { Jóvenes a } \\
\text { favor del } \\
\text { barrio } \\
\text { Compartir } \\
\text { derechos } \\
\text { Participa } \\
\text { Red joven } \\
\text { Ahuyentemos } \\
\text { el hambre } \\
\text { Jóvenes } \\
\text { lectores } \\
\text { Dinamizadores } \\
\text { deportivos } \\
\text { Proyecto } \\
\text { Cicerone } \\
\text { Mediación } \\
\text { Acciones } \\
\text { puntuales }\end{array}$ \\
\hline
\end{tabular}

En relación con las cuestiones pedagógicas que aparecen de modo repetido en los casos analizados, podemos destacar cinco bloques temáticos básicos: la vinculación del aprendizaje al currículum, la calidad educativa del servicio, las relaciones de partenariado con las entidades sociales, la evaluación del alumnado y los cambios organizativos que se requieren (Puig y otros, 2015).

Aprendizaje y currículum. Uno de los problemas pedagógicos recurrentes tienen que ver con el esfuerzo por garantizar la vinculación de los aprendizajes que proporciona o que requiere el proyecto con el currículum del curso en el que se programa la actividad de aprendizaje-servicio. Sin aprendizaje significativo, el servicio no es suficiente para justificar la actividad. Pero además los aprendizajes que se realizaran durante el proyecto han de estar relacionados directamente con aquello que el alumnado debe aprender y está previsto en el currículum.

Finalmente, los aprendizajes tienen que estar incorporados en una materia o distribuidos entra varias, pero en cualquier caso conviene que estén anclados en espacios definidos de la programación escolar.

En relación con los aprendizajes, también ha sido preocupación habitual de los centros estudiados asegurar espacios de reflexión que permitan dar sentido personal y social a la actividad y aprovechar de modo completo la experiencia vivida. Proponer actividades y buscar momentos de reflexión es una preocupación ampliamente compartida por los centros y todavía en proceso de mejora.

Servicio y necesidades. En relación al otro polo de los proyectos de aprendizaje-servicio, el servicio a la comunidad, las preocupaciones recurrentes tienen que ver con la utilidad del servicio y con su calidad educativa. En primer lugar, es fundamental idear servicios que respondan realmente a una necesidad sentida de la comunidad, lo cual convertirá la ayuda en una actividad auténtica para el alumnado y positiva de verdad para los receptores. No caer 
en servicios innecesarios es una preocupación educativa básica para que el aprendizaje servicio sea una experiencia significativa.

En segundo lugar, la actividad de servicio debe tener relación con el contenido de los aprendizajes que debe realizar el alumnado. No se trata de proponer cualquier actividad voluntaria de servicio a la comunidad, sino una que sea socialmente útil y relacionada directamente con el currículum. Finalmente, tiene que valorarse también la complejidad educativa de las tareas que se incluyen en la actividad de servicio. Deben ser actividades exigentes para el alumnado, pero accesibles a sus posibilidades personales y cognitivas.

Entidades sociales y partenariado. En los tres casos analizados, hemos visto como al comienzo del proceso de implantación del aprendizaje-servicio fue determinante la participación de alguna entidad externa que por sus demandas o por la ayuda que prestó se convirtió en un elemento desencadenante del proceso de innovación que han vivido los centros. De ahí el principio que parece regir en las actividades de aprendizaje-servicio: nada es posible en soledad. Principio que rige casi del todo en la mayoría de los proyectos. En todos ellos se necesita colaborar con una entidad social que proporcione espacios de servicio adecuados al alumnado. Esta tarea de colaboración requiere un trabajo continuado de relación, de diseño de los proyectos, de previsión de los detalles y, en definitiva, de aproximación de las respectivas culturas institucionales. Una tarea que exige dedicación y paciencia, pero que en todos los casos ha proporcionado enormes beneficios.

Evaluación del alumnado. La realidad de cada uno de los centros convierte la evaluación en una exigencia, pero en una exigencia que se lleva a cabo con intención y medios muy distintos. Resulta imposible resumir la variedad de herramientas y recursos que han desarrollado los equipos docentes, pero en todas las propuestas late la necesidad de evaluar al alumnado, de retornarle información sobre sus adquisiciones y de ayudarlo a optimizar su proceso de aprendizaje. La evaluación es un momento esencial del aprendizaje $y$, aunque los proyectos de aprendizaje-servicio tengan características peculiares, no pueden olvidar la conveniencia de llevar a cabo una evaluación formativa y acreditativa.

Las actividades de evaluación siempre encierran algo de reconocimiento. Sin embargo, el aprendizaje-servicio permite dar un paso más y organizar actos finales de reconocimiento con la participación de representantes de las entidades, receptores del servicio y, por supuesto, alumnado y profesorado. Son actos de evaluación menos académicos, menos técnicos y más cercanos a la emoción y al sentido cívico de las actividades realizadas.

Organización y logística. Una de las preocupaciones comunes a los tres centros es el tema de la organización de las actividades de aprendizajeservicio. Sin duda se trata de un 
conjunto de decisiones que cada centro deberá considerar y finalmente tomar, ya que las maneras de resolver los problemas y, en definitiva, de organizar las actividades son muy variadas. Son tan variadas que resulta imposible ni tan solo resumirlas. En todo caso, señalar que los equipos docentes deberán considera aspectos como el horario y distribución de las actividades de servicio, la organización de las salidas del centro, la preparación de las actividades que se realizan fuera del horario escolar, la gestión de los permisos y convenios correspondientes, la coordinación entre el profesorado de las distintas materias implicadas, así como otros aspectos que acaban siendo esenciales en el buen funcionamiento de las actividades de aprendizajeservicio.

Los cinco temas que han aparecido en los tres estudios realizados, tal como ya hemos afirmado, son comunes y a la vez muy variadas las soluciones que han ensayado y finalmente han adoptado. Por tanto, no es aventurado anticipar que otros centros se van a encontrar con parecidos problemas, pero que a buen seguro encontraran otras soluciones mejor adaptadas a sus circunstancias.

\section{Algunos indicios positivos}

A partir de diferentes investigaciones internacionales, algunas recogidas y resumidas por Furco (2004), ya éramos conscientes de las bondades y el impacto que produce el aprendizajeservicio a diferentes niveles de desarrollo. Sabiendo que es una tendencia generalizada, nos interesaba comprobar si en los centros estudiados se alcanzaba algún logro significativo. No entraremos en el debate sobre la evaluación de la innovación educativa, tan solo queremos comprobar si el proceso de convertir el aprendizajeservicio en un proyecto global de centro ha producido algún impacto positivo en los centros y en su alumnado. Sabemos que los datos que vamos a referenciar no son suficientes y sería necesario realizar investigaciones más completas que no han sido posibles. Sin embargo, a modo de indicio, quizás nos ayuden a dimensionar el cambio que ha supuesto para cada uno de los centros estudiados.

Mejora de la matrícula y asistencia. A Solc Nou y Fontseré les preocupaban estas dos cuestiones y ambos consiguieron notables mejoras. En el Fontseré se pasó de una preinscripción de 17 estudiantes para 60 plazas en $1^{\circ}$ de ESO a superar anualmente las 50 preinscripciones. Una vez iniciadas las transformaciones, Solc Nou incrementó también su matrícula en todos los ciclos y además consiguió reducir el abandono. Además, ambos centros han notado una reducción del ausentismo; por ejemplo en el Fontseré, de un absentismo de $16 \%$ en el curso 20042005 se ha pasado al $2 \%$ en los últimos años.

Calidad de los aprendizajes y resultados académicos. En todos los centros, el profesorado considera que ha mejorado la calidad de los aprendizajes que logra su alumnado. En el caso del Fontseré se ha pasado de una tasa de graduados en ESO del 45 $\%$ al $80 \%$. Asimismo, en el mismo 
centro, en las pruebas de competencias básicas se pasó de una media entre 1,5 y 3 según el área a situarse entre 5,5 y 6,8 .

Mejora del clima de centro y de las condiciones de aprendizaje.

Nuevamente, el profesorado de los tres centros constata mejoras ostensibles en el clima de convivencia y satisfacción del alumnado. En el caso del Fontseré se pasó de más de cuarenta partes disciplinarios al año a menos de tres por curso. Una cambio fundamental para mejorar los aprendizajes y la vida cotidiana del centro.

Reconocimiento de los centros y participación ciudadana del alumnado. Hemos podido constatar que los cambios han llevado a que los centros reciban diferentes reconocimientos externos: premios, oportunidades para presentar su experiencia en jornadas o participar en espacios académicos. Asimismo, tanto el alumnado del Xaloc como del Fontseré, pasada la etapa escolar, se interesaban por implicarse en actividades de voluntariado social, por participar en esplais o en las entidades de cultura popular del barrio.

Como último apunte, nos gustaría destacar, que dada la realidad de los centros con los que hemos podido trabajar, ha sido interesante ver como respondía el aprendizaje servicio en cada caso como herramienta útil para un enfoque inclusivo en la educación especial (EE. Xaloc), para romper con los prejuicios y cambiar la imagen de los y las jóvenes en situación de exclusión (INS Fontseré), y para buscar nuevos enfoques más vivenciales para la educación en valores en entornos más profesionalizadores (Solc Nou).

\section{Propuestas para generalizar el aprendizaje-servicio en un centro}

A modo de síntesis de lo que hemos presentado hasta ahora, vamos a proponer un conjunto de recomendaciones que, en los casos analizados, han ayudado a idear proyectos educativos que giran en torno al aprendizaje-servicio. Con una frecuencia de al menos una vez cada año para todo su alumnado, el aprendizaje-servicio se ha convertido en estos centros en un elemento esencial de su línea educativa. A continuación veremos algunas propuestas que han contribuido a que equipos docentes que no conocían el aprendizaje-servicio, lo ofrezcan con frecuencia a todo su alumnado. Las líneas de acción que hemos recogido surgen del análisis de los procesos que han seguido los centros estudiados, se trata de propuestas que se repiten en todos los casos, que han sido relevantes para alcanzar los objetivos que se perseguían y que pueden ayudar a que otros centros educativos ofrezcan múltiples actividades de aprendizajeservicio a su alumnado. Es decir, conviertan el aprendizaje-servicio en un nota de identidad del centro: lo consideren parte de su filosofía, lo declaren en su proyecto educativo, lo incluyan en sus planes docentes y acabe implicando a la mayoría de su profesorado.

Las recomendaciones que se van a enumerar se dirigen principalmente a los primeros responsables de aplicarlas, 
Ios equipos docentes y sus direcciones, aunque naturalmente implican también a todos los participantes en las actividades de aprendizaje-servicio: profesorado, alumnado, miembros de las entidades colaboradoras, mediadores territoriales, receptores del servicio y familias. Veamos las siguientes propuestas levemente explicadas.

Construir un reto. A partir de problemas acuciantes que viven los centros o, en caso de no haberlos, a partir de la voluntad de mejorar sus resultados, los equipos educativos deben definir y compartir un reto que dibuje con claridad lo que se desea alcanzar; una finalidad que actúa como horizonte y como motivación.

Detectar oportunidades. Cuando se tiene un problema que resolver, lo normal es buscar por todas partes ideas que ayuden a encauzarlo. Eso es lo que hicieron nuestros centros y una de las ideas que encontraron fue el aprendizaje-servicio, que resultó ser una metodología adecuada a sus necesidades. Ahora que ya sabemos de la bondad de esta propuesta, la principal tarea es buscar actividades que ya se realizan en el centro y son cercanas al aprendizaje-servicio, profesorado dispuesto a implicarse, espacios curriculares donde acoger tales propuestas $y$, finalmente, disponer todo ello de manera que se logren realizar las primeras experiencias.

Ejercer el liderazgo. Un proceso de innovación como el que supone generalizar el aprendizaje-servicio requiere un liderazgo individual o colectivo claro, que se ponga al servicio del equipo, que tenga visión de futuro y que permita definir conjuntamente un proyecto motivador. Un liderazgo que contribuya a fijar el reto y a vivirlo en positivo, que sea capaz de respetar y aceptar a todos los miembros del grupo, que logre sacar lo mejor de cada uno de ellos, facilite su trabajo y atienda con calidez y firmeza cada una de las situaciones que se presenten. Por último, debe ser un liderazgo capaz de distribuir responsabilidades, evaluar los resultados conjuntamente y ayudar a que todos sean capaces de alcanzar con éxito las tareas encomendadas.

Crear equipo. Sin construir un nosotros y darse las herramientas para el trabajo colectivo no es posible impulsar una innovación de calado. Es necesario, primero, reconocer las competencias de cada uno de los miembros del equipo $y$, luego, dar la palabra y organizar de modo habitual espacios de diálogo en los que sea posible llevar a cabo una reflexión profunda y reposada. Espacios que no son simplemente las reuniones habituales de funcionamiento del centro, sino momentos de creación y de apropiación personal de los pasos que va dando el grupo. Finalmente, ese trabajo dibujará un proyecto común que ayudará a guiar el trabajo cotidiano.

Buscar socios. Un centro educativo tan solo con sus fuerzas y posibilidades es muy difícil que pueda llevar a cabo una actividad de aprendizaje-servicio y menos generalizarla a todo su alumnado. La contribución de las entidades sociales de la comunidad es 
esencial para definir actividades de servicio, abrir espacio de trabajo, aportar conocimientos al aprendizaje del alumnado, acordar fórmulas de colaboración y, en definitiva, alcanzar acuerdos beneficiosos para los centros y para las entidades.

Idear un proyecto y mejorar su calidad pedagógica. La colaboración entre el equipo docente y los miembros de las entidades sociales permitirá idear una propuesta de aprendizaje-servicio interesante, formativa y sostenible. Sin embargo, su proceso de mejora no concluye en este momento inicial. Por el contrario, conviene evaluar con frecuencia la calidad pedagógica de cada una de las actividades de aprendizaje-servicio. Una propuesta articula un conjunto de dinamismos que se pueden implementar cada vez de un modo algo mejor.

\section{Buscar complicidades con el alumnado} y sus familias. Los primeros y más importantes cómplices de un centro que desee implantar el aprendizajeservicio de forma amplia es su alumnado. Y no solo por su implicación en cada una de las propuestas que se les ofrezca, sino también por su participación en el sostén del conjunto del proyecto y por considerar el aprendizaje-servicio una actividad que les llena de orgullo. Asimismo, conviene implicar a las familia, algo que se consigue dándoles información, explicando qué es el aprendizajeservicio y por que motivos se programa, mostrando el compromiso del centro con el aprendizaje-servicio y si es necesario pidiéndoles su ayuda. Además de alumnado y familias, puede ser muy interesante contar con la complicidad de las administraciones, local y educativa, así como con instituciones dedicadas a la promoción del aprendizaje-servicio.

Evaluar la adecuación de las actividades al proyecto de centro. Además de mejorar la calidad pedagógica de cada experiencia, es necesario también evaluarlas para comprobar si alcanzan los objetivos propuestos, si conviene modificar algunos aspectos para hacerlos mejores, o si lo más adecuado es cerrarlos y pensar en nuevas alternativas. Los propuestas de aprendizaje-servicio no son eternas y lo que un día sirvió para alcanzar los objetivos pedagógicos propuestos, puede que ahora por múltiples motivos no sean de utilidad. No es posible imaginar un proceso de implantación masiva del aprendizaje-servicio sin cambios, substituciones y nuevas propuestas.

Celebrar y reconocer los éxitos. El equipo de profesorado y por supuesto los alumnos deben recibir todo el refuerzo positivo que sea posible. De este modo se reconoce su esfuerzo, se les empodera en las tareas que les son propias y se les motiva a continuar con el esfuerzo. Conviene realizar al final de cada actividad actos de reconocimiento con la participación de todos los implicados y, por otra parte, es conveniente que el equipo de profesorado reciba reconocimiento público de su esfuerzo.

Este conjunto de recomendaciones derivan del análisis de los tres centros 
estudiados y, por lo tanto, tienen cierta plausibilidad y es fácil que puedan ser una buena inspiración para que otros centros sigan, si así lo desean, un camino parecido. Sin embargo, conviene estar abierto a otras estrategias que permitan alcanzar objetivos parecidos por caminos distintos. El horizonte que aquí se considera es la implantación del aprendizaje-servicio en un centro educativo para que cada año pueda implicarse todo su alumnado. Pero el camino para conseguirlo no debe estar cerrado a innovaciones en el modo alcanzar tal objetivo.

\section{Referencias bibliográficas}

Bär, B. (2016a). Trencar prejudicis $i$ millorar resultats. L'aprenentatge servei a I'IES Eduard Fontseré. Disponible en http://www.aprenentatgeservei.org/intr a/aps/documents/Quadern\%20APS Tre ncant\%20prejucidis Fontsere.pdf

Bär, B. (2016b). Aprenentatge servei, un enfocament inclusiu a l'educació especial. L'etapa 16-20 a l'Escola d'Educació Especial Xaloc de Sabadell. Disponible en http://www.aprenentatgeservei.org/intr a/aps/documents/Quadern\%20APS Xal oc.pdf

Barber, B.R. (2000). Un lugar para todos. Barcelona, Paidós.

Carmona, A.; Bär, B. y Puig, J. (2016). Educar con sentido. Aprendizaje servicio en la Escuela Solc Nou. Barcelona: Edebé. Disponible en http://www.aprenentatgeservei.org/intr a/aps/documents/Libro\%20Educar\%20 con $\% 20$ sentido Solc $\% 20$ Nou.pdf

Dewey, J. (1967). Experiencia y educación. Buenos Aires: Losada.

Folgueiras, P.; Luna, E. y Puig, G. (2013). Aprendizaje y Servicio: estudio del grado de satisfacción de estudiantes universitarios. Revista de Educación, 362, 159-185.

Furco, A. (2004). El impacto educacional del aprendizaje-servicio. University of California-Berkeley. Disponible en. http://roserbatlle.net/wpcontent/uploads/2009/03/resultatsinvestigacio-aps-furco-modo-decompatibilidad.pdf

Puig, J. (2003). Prácticas morales. Barcelona: Paidós.

Puig, J. (Coord.) (2015). 11 ideas clave. ¿Cómo realitzar un proyecto de aprendizaje Servicio? Barcelona: Graó.

Puig, J.; Batlle, R.; Bosch, C. y Palos, J. (2006). Aprenentatge servei. Educar per a la ciutadania. Barcelona:

Octaedro.

Taylor, S.J. y Bogdan, R. (1987). Introducción a los métodos cualitativos de investigación. Barcelona: Paidós. 